\title{
Joint impacts of relaying scheme and wireless power transfer in multiple access of cellular networks
}

\author{
Anh-Tu Le ${ }^{1}$, Dinh-Thuan Do ${ }^{1}$ \\ ${ }^{1}$ Faculty of Electronics Technology, Industrial University of Ho Chi Minh City, Vietnam
}

\begin{tabular}{|c|c|}
\hline Article Info & ABSTRACT \\
\hline Article history: & \multirow{8}{*}{$\begin{array}{l}\text { This paper considers ergodic capacity of energy harvesting (EH) based cellular net- } \\
\text { works. Such a network employs non-orthogonal multiple access (NOMA) together } \\
\text { with relaying scheme to serve two far users. In this system model, relay is facili- } \\
\text { tated power splitting (PS) protocol to implement energy harvesting (EH). To examine } \\
\text { capacity, expressions of signal to noise ratio (SNR) need be computed to achieve ca- } \\
\text { pacity. Power allocation factors are different for two users in such system and hence } \\
\text { performance gap happens to distinguish requirements for separated users. It can be } \\
\text { confirmed that the proposed paradigm exhibits maximal achievable capacity in some } \\
\text { scenarios of setting parameters. To confirm exactness of the analytical expressions } \\
\text { and show advantages of the proposed EH-NOMA, simulation results are performed in } \\
\text { terms of ergodic capacity. }\end{array}$} \\
\hline Received Oct 16, 2020 & \\
\hline Revised Jan 7, 2021 & \\
\hline Accepted Feb 16, 2021 & \\
\hline Keywords: & \\
\hline & \\
\hline JOMA & \\
\hline Energy harvesting & \\
\hline
\end{tabular}

This is an open access article under the CC BY-SA license.



\section{Corresponding Author:}

Dinh-Thuan Do

Faculty of Electronics Technology

Industrial University of Ho Chi Minh City

12 Nguyen Van Bao, Go Vap Dist., Ho Chi Minh City, Vietnam

Email: dodinhthuan@iuh.edu.vn

\section{INTRODUCTION}

Considering as the potentials to implement infrastructure in the fifth generation (5G) cellular system, higher spectral efficiency benefits from the non-orthogonal multiple access (NOMA) technique to serve massive connections [1], [2]. To overcome disadvantages of the traditional orthogonal multiple access (MA) technique, the power domain is employed to require NOMA to provide MA in cellular networks [3]. To perform functions of NOMA, the signals of multiple users are multiplexed in the power domain at the base station (BS), i.e. superposition coding (SupC) is designed at transmitters. At the receivers, to restrain the inter-user interference, it is required to employ successive interference cancellation (SIC). In addition to power-reuse, NOMA provides extra advantage in term of user fairness as distinctive feature of this scheme [4]. In this MA scheme, users with worse channel conditions need allocate more power, while users with higher channel gains only require less power. By implementing NOMA communication system, it can be achieved improved trade-off between user fairness and system performance. For example, higher throughput and faster traffic are two advantages of NOMA compared with the conventional orthogonal MA protocols.

Together with NOMA, this technique has more benefits as combining with cooperative transmission scenarios and further improvement of the reliability in NOMA is obtained [5]-[8]. In cooperative manner, users and relays can cooperate to combat channel fading and improve the performances, namely new scheme as cooperative NOMA (C-NOMA) networks. At the receivers, the maximal-ratio combining (MRC) needs to combine the two independent signals received at two links including the direct and relaying. As a result, C-NOMA 
provides ability of the diversity techniques. Thus, C-NOMA exhibits better qualities of services (Qos) criteria and the system can be improved significantly. Recently, C-NOMA has been intensively studied. Generally, various aspects of C-NOMA were introduced. The reliability in transmission and improved performance can be achieved as in recent works [9]-[15]. In recent works [13], [15], the authors indicated advantages of NOMA can be achieved in device to device networks. Regarding energy harvesting [16-19], results exhibits wireless power transfer as solution to prolong lifetime of network and applications of NOMA. The closed forms of the metrics, such as the in terms of outage probability, throughput. In most of works, the target rates are main factor affecting to outage behavior and they derived expressions to evaluate the performance of the NOMA scheme.

Nowadays, power domain based NOMA is widely studied in works, such as [20], [21], [22]. They introduced system adopting orthogonal frequency division multiplexing in the sub-channel transmission of PDNOMA [23]. The multi-user detection uses SIC technology at the receiver to delete interference among users and such Non-orthogonal transmission still remains its performance. The authors in [24] examined performance of a hybrid NOMA-based wireless system. In this system, to transmit on the uplink, users harvest wireless power from the received downlink signals. To improve the spectral efficiency by exploiting the spatial domainJ. W. Kim et al. proposed an integration of NOMA and generalized space shift keying [26].

Motivated by recent works [16], [17], [18], this paper considers a new the fixed power allocation scheme and relay harvests power and acts as relay with respect to implement cooperative EH-NOMA protocol. Such relay is designed to introduce performance gaps to representative how well the weak user and strong user operate in various scenarios.

\section{SYSTEM MODEL AND SINR COMPUTATIONS}

\subsection{System model}

By exploring wireless power transfer, relay in Figure 1 is able to harvesting power from a base station (BS). In this scenario, two destinations $\left(U_{1}, U_{2}\right)$ with a power splitting-based EH relay [18]-[21] is studied. We denote channel gains $h_{a b}$ for link from node $a$ to node $b$ and it falls into exponential distribution with means $\lambda_{a b}$. The channels are quasistatic, i.e. the channels remains constant over one transmission time while different values for these channels over different transmission times. Relay has two kinds, Amplify-and-Forward (AF) or Decode-and-Forward (DF) relaying and it suitable for relevant applications. $T$ is denoted as the whole transmission time. $T$ is divided into two transmission phases. Regarding EH function, it uses only the harvested power during the first transmission phase to transmit during the second transmission phase. Regarding the transmit power of the BS, $P_{S}$ is transmit power. To serve two users, $x_{1}$ and $x_{2}$ are the messages intending to send to the weak user $U_{1}$ and the strong user $U_{2}$. We denote $a_{1}$ and $a_{2}$ as the power allocation coefficients in NOMA scheme. It is strict required: $a_{1}>a_{2}$ with $a_{1}+a_{2}=1$.

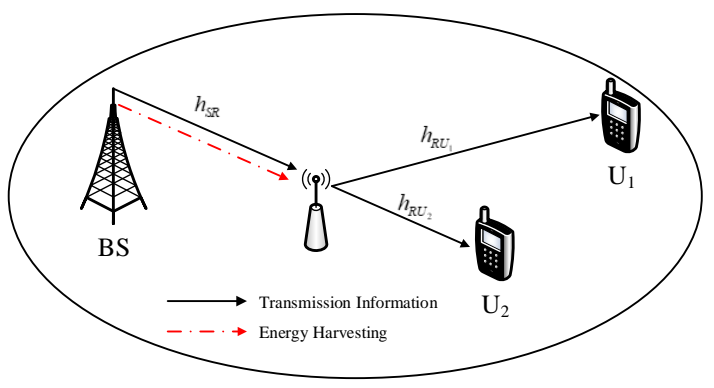

Figure 1. Wireless power transfer in NOMA.

The relay is able to harvest wireless energy to serve transmission in the next phase, it need be computed the received signal at the relay $R$ as

$$
y_{R}=\sqrt{(1-\beta)} h_{S R} \mu(x)+n_{R}
$$

where $\mu(x)=\left(\sqrt{a_{1} P_{S}} x_{1}+\sqrt{a_{2} P_{S}} x_{2}\right)$ is mixture signal, $n_{R}$ is known as the additive white Gaussian noise (AWGN) with variance $N_{0}$. More importantly, by controlling transmit power $P_{S}$ at the BS with arbitrary coeffi- 
cient denoted by $\beta(0<\beta<1)$, amount of harvested power varies significantly and hence system performance needs be guaranteed.

by $[27]$

Although litter amount of harvested energy, the relay benefits from such energy, and it is formulated

$$
P_{h}=\beta P_{S}\left|h_{S R}\right|^{2} \text {. }
$$

We denote $\left|h_{S R}\right|^{2}$ as variable $X_{a}$ In the next section, the first metric needs be considered, i.e. the signal-tointerference-plus-noise ratio (SINR) and signal-to-noise ratio (SNR) are then used in further metrics.

\subsection{SINR computations}

Together with performing successive interference cancellation (SIC) in NOMA, SINR, SNR can be determined to detect signal $x_{1}, x_{2}$ respectively at $\mathrm{R}$ and they can be given as

$$
\begin{aligned}
\gamma_{S R 1} & =\frac{(1-\beta) a_{1} P_{S} X_{a}}{(1-\beta) a_{2} P_{S} X_{a}+N_{0}} \\
& =\frac{(1-\beta) a_{1} \rho X_{a}}{(1-\beta) a_{2} \rho X_{a}+1},
\end{aligned}
$$

and

$$
\gamma_{S R 2}=(1-\beta) a_{2} \rho X_{a},
$$

Considering the main impact on outage performance $\rho=\frac{P_{S}}{N_{0}}$ is denoted as transmit SNR and it is computed at the BS.

In order to detect $x_{1}$ at user $U_{1}$, the corresponding SINR is expressed by

$$
\gamma_{R U_{1}}=\frac{\beta \rho a_{1} X_{b} X_{a}}{\beta \rho a_{2} X_{a} X_{b}+1}
$$

where $\left|h_{S U_{1}}\right|^{2}$ is denoted as variable $X_{b}$. Similarly, the SINR to detect $x_{2}$ at user $U_{2}$ is computed by

$$
\gamma_{R U_{2,1}}=\frac{\beta \rho a_{1}\left|h_{R U_{2}}\right|^{2} X_{a}}{\beta \rho a_{2} X_{a}\left|h_{R U_{2}}\right|+1} .
$$

SNR to to detect $x_{2}$ is only determined after SIC implementation at $U_{2}$, and it is formulated as

$$
\gamma_{R U_{2}}=\beta \rho a_{2}\left|h_{R U_{2}}\right|^{2}\left|h_{S R}\right|^{2} .
$$

\subsection{Decode-and-forward relaying}

DF relaying mode is also known as popular strategy to extend coverage of wireless network under the context of EH-NOMA using the end-to-end SNR from the BS to user $U_{1}$ can be expressed as

$$
\begin{aligned}
\gamma_{U_{1}}^{D F} & =\min \left(\gamma_{S R 1}, \gamma_{R U_{1}}\right) \\
& =\min \left(\frac{(1-\beta) a_{1} \rho X_{a}}{(1-\beta) a_{2} \rho X_{a}+1}, \frac{\beta \rho a_{1} X_{b} X_{a}}{\beta \rho a_{2} X_{a} X_{b}+1}\right)
\end{aligned}
$$

It is worth noting that such min function is maximized when all of its argument becomes equal. As a result, the following optimal value of $\beta$ is found to achieve maximal instantaneous rate as

$$
\beta_{D F, U_{1}}^{*}=\frac{1}{X_{b}+1}
$$

For DF case, the SNR from the BS to $U_{2}$ can be computed as

$$
\begin{aligned}
\gamma_{U_{2}}^{D F} & =\min \left(\gamma_{S R 2}, \gamma_{R U_{2}}\right) \\
& =\min \left((1-\beta) a_{2} \rho X_{a}, \beta \rho a_{2}\left|h_{R U_{2}}\right|^{2} X_{a}\right) .
\end{aligned}
$$

Similarly, the optimal value of $\beta$ can be obtained to achieve optimal instantaneous rate as

$$
\beta_{D F, U_{2}}^{*}=\frac{1}{\left|h_{R U_{2}}\right|^{2}+1} .
$$




\subsection{Amplify-and-forward relaying}

For AF- based EH-NOMA, the received SNR at the $U_{1}$ can be formulated by [Eq.4,[22]]

$$
\begin{aligned}
\gamma_{U_{1}}^{A F} & \simeq \frac{\gamma_{S R 1} \gamma_{R U_{1}}}{\gamma_{S R 1}+\gamma_{R U_{1}}} \\
& \simeq \frac{(1-\beta) \beta \rho a_{1} X_{b} X_{a}}{2(1-\beta) \beta \rho a_{2} X_{a} X_{b}+(1-\beta)+\beta X_{b}}
\end{aligned}
$$

Similarly, the SINR can be obtained at $U_{2}$ as

$$
\gamma_{U_{2}}^{A F} \simeq \frac{(1-\beta) \beta \rho a_{2}\left|h_{R U_{2}}\right|^{2} X_{a}}{(1-\beta)+\beta\left|h_{R U_{2}}\right|^{2}}
$$

The following optimal value of $\beta$ in AF scenario is expressed by

$$
\beta_{A F, U_{i}}^{*}=\frac{1}{\left|h_{R U_{i}}\right|+1}
$$

\section{ERGODIC CAPACITY}

\subsection{Decode-and-Forward relaying}

3.1.1. The ergodic capacity at $U_{1}$

The analytical expression can be obtained for ergodic capacity [2]

$$
\begin{aligned}
C_{U_{1}}^{D F} & =\mathrm{E}\left[\frac{1}{2} \log _{2}\left(1+\gamma_{U_{1}}^{D F}\right)\right] \\
& =\frac{1}{2 \ln 2} \int_{0}^{\infty} \frac{1-F_{\gamma_{U_{1}}^{D F}}(x)}{1+x} d x
\end{aligned}
$$

where $\mathrm{E}[\bullet]$ denotes the expectation over random variable. Then, we can write $F_{\gamma_{U_{1}}^{D F}}(x)$ as

$$
\begin{aligned}
F_{\gamma_{U_{1}}^{D F}}(x) & =\operatorname{Pr}\left(\gamma_{D_{1}}^{D F}<x\right) \\
& =1-\operatorname{Pr}\left(X_{a}>\frac{x\left(\left|h_{1}\right|^{2}+1\right)}{\rho\left(a_{1}-x a_{2}\right)\left|h_{1}\right|^{2}}\right)
\end{aligned}
$$

It then can be written as

$$
\begin{aligned}
F_{\gamma_{U_{1}} F}(x) & =\int_{0}^{\infty} f_{\left|h_{1}\right|^{2}}(z) \int_{0}^{\frac{x(z+1)}{\rho\left(a_{1}-x a_{2}\right) z}} f_{X_{a}}(y) d y d x \\
& =1-\frac{1}{\lambda_{1}} \int_{0}^{\infty} e^{-\frac{x(z+1)}{\rho\left(a_{1}-x a_{2}\right) z \lambda_{S R}}-\frac{z}{\lambda_{1}}} d x \\
& =1-2 e^{-\frac{\gamma_{t h 1}}{\left(a_{1}-x a_{2}\right) \rho \lambda_{S R}}} \sqrt{\frac{\gamma_{t h 1}}{\left(a_{1}-x a_{2}\right) \rho \lambda_{S R} \lambda_{1}}} \\
& \times K_{1}\left(2 \sqrt{\frac{\gamma_{t h 1}}{\left(a_{1}-x a_{2}\right) \rho \lambda_{1} \lambda_{S R}}}\right)
\end{aligned}
$$

As a result, ergodic capacity in optimal scenario and $\mathrm{DF}$ mode for $U_{1}$ is given by

$$
\begin{aligned}
C_{U_{1}}^{D F} & =\frac{1}{\ln 2} \int_{0}^{a_{1} / a_{2}} \frac{e^{-\frac{\lambda_{R U_{1} x}}{\left(a_{1}-x a_{2}\right) \rho}}}{1+x} \\
& \times \sqrt{\frac{x \lambda_{R U_{1} \lambda_{S R}}}{\left(a_{1}-x a_{2}\right) \rho} K_{1}}\left(2 \sqrt{\frac{x \lambda_{S R} \lambda_{R U_{1}}}{\left(a_{1}-x a_{2}\right) \rho}}\right) d x
\end{aligned}
$$




\subsubsection{The ergodic capacity at $U_{2}$}

Similarly, ergodic capacity is expressed for $U_{2}$ in DF mode as

$$
\begin{aligned}
C_{U_{2}}^{D F} & =\mathrm{E}\left\{\frac{1}{2} \log _{2}\left(1+\gamma_{U_{1}}^{D F}\right)\right\} \\
& =\mathrm{E}\left(\frac{e^{\frac{\lambda_{S R}\left(X_{b}+1\right)}{a_{2} \rho X_{b}}} E_{1}\left(\frac{\lambda_{S R}\left(X_{b}+1\right)}{a_{2} \rho X_{b}}\right)}{2 \ln 2} d X_{b}\right)
\end{aligned}
$$

where $E_{1}($.$) is the exponential integral. Because, it is difficult to solve (19) in closed-form, so we can use the$ following approximation for the exponential integral [24]

$$
E_{1}(x) \simeq 4 \sqrt{2} \pi a_{N} a_{I} \sum_{n=1}^{N+1} \sum_{i=1}^{I+1} \sqrt{b_{n}} e^{-4 b_{n} b_{i} x}
$$

where $a_{N}=\frac{1}{2 N+2}, a_{I}=\frac{1}{2 I+2}, b_{n}=\frac{\cot \left(\theta_{n-1}\right)-\cot \left(\theta_{n}\right)}{(N+1)^{-1} \pi}, b_{i}=\frac{\cot \left(\theta_{i-1}\right)-\cot \left(\theta_{i}\right)}{(I+1)^{-1} \pi}, \theta_{0}=0, \theta_{n}=\frac{\pi n}{2 N+2}$, $\theta_{i}=\frac{\pi i}{2 I+2}$.

Hence, $C_{U_{2}}^{D F}$ can be rewritten as

$$
\begin{aligned}
C_{U_{2}}^{D F} & =\frac{2 \sqrt{2}}{\ln 2} \pi a_{N} a_{I} \sum_{n=1}^{N+1} \sum_{i=1}^{I+1} \sqrt{b_{n}} \\
& \times \int_{0}^{\infty} \lambda_{R U_{1}} e^{-\lambda_{R U_{1}} X_{b}} e^{-\varepsilon \frac{\left(x_{b}+1\right)}{X_{b}}} d X_{b}
\end{aligned}
$$

where $\varepsilon=\frac{4 b_{n} b_{i} \lambda_{S R}}{a_{2} \rho}-\frac{\lambda_{S R}}{a_{2} \rho}$. Based on $[23,3.324 .1]$, the capacity of $U_{2}$ can be expressed as

$$
C_{U_{2}}^{D F}=\frac{2 \sqrt{2}}{\ln (2)} \pi a_{N} a_{I} \sum_{n=1}^{N+1} \sum_{i=1}^{I+1} \sqrt{\lambda_{R U_{1}} b_{n}} e^{-\varepsilon} \sqrt{\varepsilon} K_{1}\left(2 \sqrt{\varepsilon \lambda_{R U_{1}}}\right)
$$

\subsection{Amplify-and-forward relaying}

3.2.1. The ergodic capacity at $U_{1}$

We have formula for examining ergodic capacity at $U_{1}$ in AF mode as

$$
\begin{aligned}
C_{U_{1}}^{A F} & =\mathrm{E}\left[\frac{1}{2} \log _{2}\left(1+\gamma_{U_{1}}^{A F}\right)\right] \\
& =\frac{1}{2 \ln 2} \int_{0}^{\infty} \frac{1-F_{\gamma_{U_{1}}^{A F}}(x)}{1+x} d x .
\end{aligned}
$$

It is worth noting that $F_{\gamma_{U_{1}}^{A F}}(x)$ is written as

$$
\begin{aligned}
F_{\gamma_{U_{1}}^{A F}}(x) & =\operatorname{Pr}\left(\hat{\gamma}_{U_{2}}^{A F}<x\right) \\
& =\operatorname{Pr}\left(\frac{\rho a_{2} X_{a}\left|h_{R U_{2}}\right|^{2}}{\left(1+\left|h_{R U_{2}}\right|\right)^{2}}<x\right) \\
& =\operatorname{Pr}\left(X_{a}<\frac{x\left(1+\left|h_{R U_{2}}\right|\right)^{2}}{\rho a_{2}\left|h_{R U_{2}}\right|^{2}}\right)
\end{aligned}
$$


Using the CDF of $X_{a}$ and the approximation $e^{-\frac{2 \lambda_{S R} \gamma_{t h 2}}{\rho a_{2} \sqrt{z}}}=1-\frac{2 \lambda_{S R} \gamma_{t h 2}}{\rho a_{2} \sqrt{z}}$ we can write

$$
\begin{aligned}
F_{\gamma_{U_{1}}^{A F}}(x) & =1-\lambda_{R U_{2}} \int_{0}^{\infty} e^{-\frac{\lambda_{S R} x(1+\sqrt{z})^{2}}{\rho a_{2} z}} e^{-\lambda_{R U_{2}} z} d z \\
& \simeq 1-\int_{0}^{\infty} \lambda_{R U_{2}} e^{-\frac{\lambda_{S R^{x}}}{\rho a_{2}}} e^{-\frac{\lambda_{S R_{2} x}}{\rho a_{2} z}-\lambda_{R U_{2}} z} d z \\
& +\frac{2 \lambda_{S R} \lambda_{R U_{2}} x e^{-\frac{\lambda_{S R^{2}}}{\rho a_{2}}}}{\rho a_{2}} \int_{0}^{\infty} \frac{1}{\sqrt{z}} e^{-\frac{\lambda_{S R_{2}}}{\rho a_{2} z}-\lambda_{R U_{2}} z} d z
\end{aligned}
$$

To continue compute it, $F_{\gamma_{U_{1}}^{A F}}(x)$ is rewritten as

$$
\begin{aligned}
F_{\gamma_{U_{1}}^{A F}}(x) & \simeq 1-2 e^{-\frac{\lambda_{S R^{x}}}{\rho a_{2}}} \sqrt{\frac{\lambda_{S R} \lambda_{R U_{2} x}}{\rho a_{2}}} K_{1}\left(2 \sqrt{\frac{\lambda_{S R} \lambda_{R U_{2}} x}{\rho a_{2}}}\right) \\
& +e^{-\frac{\lambda_{S R_{2} x}}{\rho a_{2}}} \frac{4 x \lambda_{S R} \lambda_{R U_{2}}^{2}}{\rho a_{2}}\left(\frac{x \lambda_{S R}}{\rho a_{2} \lambda_{R U_{2}}}\right)^{1 / 4} K_{1 / 2}\left(2 \sqrt{\frac{x \lambda_{S R} \lambda_{R U_{2}}}{\rho a_{2}}}\right)
\end{aligned}
$$

After performing some manipulations, it can be obtained below result

$$
\begin{aligned}
C_{U_{1}}^{A F} & =\frac{1}{\ln 2} \int_{0}^{a_{1} / 2 a_{2}} \frac{1}{1+x}\left(e^{-\frac{\lambda_{S R^{x}}}{\rho\left(a_{1}-2 x a_{2}\right)}} \sqrt{\frac{x \lambda_{S R} \lambda_{R U_{1}}}{\rho\left(a_{1}-2 x a_{2}\right)}}\right. \\
& \times K_{1}\left(2 \sqrt{\frac{x \lambda_{S R} \lambda_{R U_{1}}}{\rho\left(a_{1}-2 x a_{2}\right)}}\right)-e^{-\frac{\lambda_{S R} x}{\rho\left(a_{1}-2 x a_{2}\right)}} \frac{2 x \lambda_{S R} \lambda_{R U_{1}}^{2}}{\rho\left(a_{1}-2 \gamma_{t h 1} a_{2}\right)} \\
& \left.\times\left(\frac{x \lambda_{S R}}{\rho\left(a_{1}-2 x a_{2}\right) \lambda_{R U_{1}}}\right)^{1 / 4} K_{1 / 2}\left(2 \sqrt{\frac{x \lambda_{S R} \lambda_{R U_{1}}}{\rho\left(a_{1}-2 x a_{2}\right)}}\right)\right) d x
\end{aligned}
$$

\subsubsection{The ergodic capacity at $U_{2}$}

Similarly, it can be illustrated ergodic capacity for $U_{2}$ as

$$
\begin{aligned}
C_{U_{2}}^{A F} & =\mathrm{E}\left(\frac{1}{2} \int_{0}^{\infty} \log \left(1+\frac{\rho a_{2}\left|h_{R U_{2}}\right|^{2} X_{a}}{\left(\left|h_{R U_{2}}\right|+1\right)^{2}}\right)\right. \\
& \left.\times \lambda_{S R} e^{-\lambda_{S R} X_{a}} d X_{a}\right) \\
& =\frac{1}{\ln 2} \int_{0}^{\infty} E_{1}\left(\frac{\lambda_{S R}\left(\left|h_{R U_{1}}\right|+1\right)^{2}}{a_{2} \rho X_{b}}\right) \\
& \times e^{\frac{\lambda_{S R}\left(\left|h_{R U_{1}}\right|+1\right)^{2}}{a_{2} \rho X_{b}}} \lambda_{S R} e^{-\lambda_{S R} X_{a}} d\left|h_{S R}\right|^{2}
\end{aligned}
$$

Finally, in AF mode, we can computed ergodic capacity for $U_{2}$ as

$$
\begin{aligned}
C_{U_{2}}^{A F} & =\frac{2 \sqrt{2}}{\ln (2)} \pi a_{N} a_{I} \sum_{n=1}^{N+1} \sum_{i=1}^{I+1} \sqrt{b_{n}} e^{-\varepsilon} \\
& \times \sqrt{\frac{\lambda_{R U_{1}}}{\varepsilon+\lambda_{R U_{1}}} \varepsilon \lambda_{R U_{1}}} K_{1}\left(2 \sqrt{\varepsilon\left(\varepsilon+\lambda_{R U_{1}}\right)}\right)
\end{aligned}
$$




\section{SIMULATION RESULT}

The simulation model is based on Figure 1, and we assume fixed power allocation factors assigned for two NOMA users, $a_{1}=0.9, a_{2}=0.1$. In the simulations, we set $\lambda_{S R}=\lambda_{R U_{2}}=1, \lambda_{R U_{1}}=2$.

In Figure 2, we investigate the impact of $\mathrm{AF}$ and $\mathrm{DF}$ relaying mode on ergodic capacity performance of EH-NOMA system. It can be seen that ergodic of user $U_{1}, U_{2}$ at mode DF is better than that of AF mode. However, user $U_{2}$ provides higher ergodic capacity compared with that of user $U_{1}$. The main reason is that different power allocation factors are used for two users. The second reason is related to decoding procedure at each user is differ.

Figure 3 depicts how strong channel of link from the BS to relay make influence to ergodic capacity. It can be intuitively seen that strong channel gain results in weaker channel of link from relay to destinations and such situation exhibits worse performance. Figure 4 indicates opposite trends for two users regarding power allocation factors. Therefore, controlling power factor $a_{1}$ guarantees fairness among two users in EH-NOMA system.

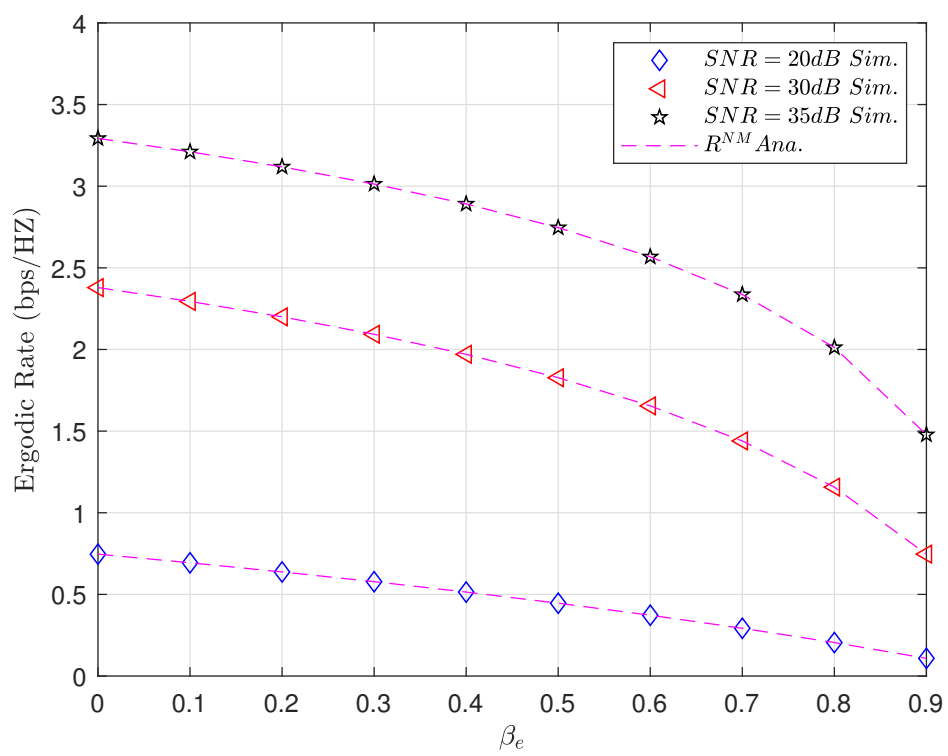

Figure 2. Ergodic capacity performance of two users versus SNR with $R_{2}=0.5$.

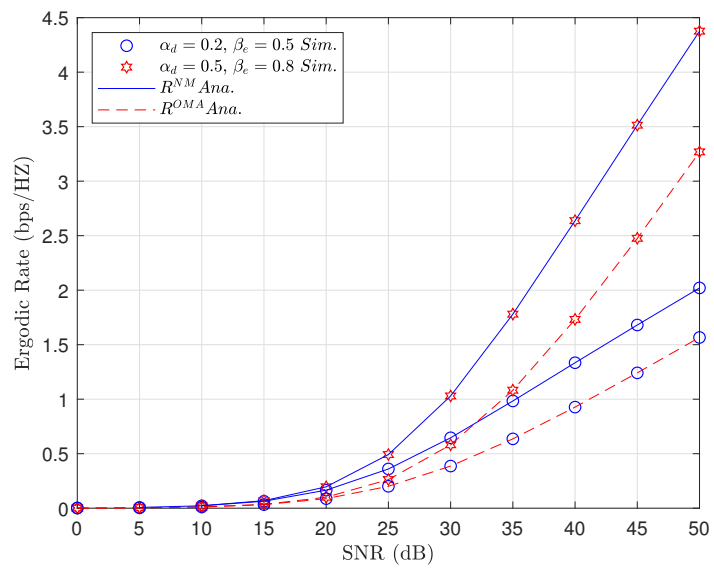

Figure 3. Ergodic capacity performance of two users versus channel gain $\lambda_{S R}$

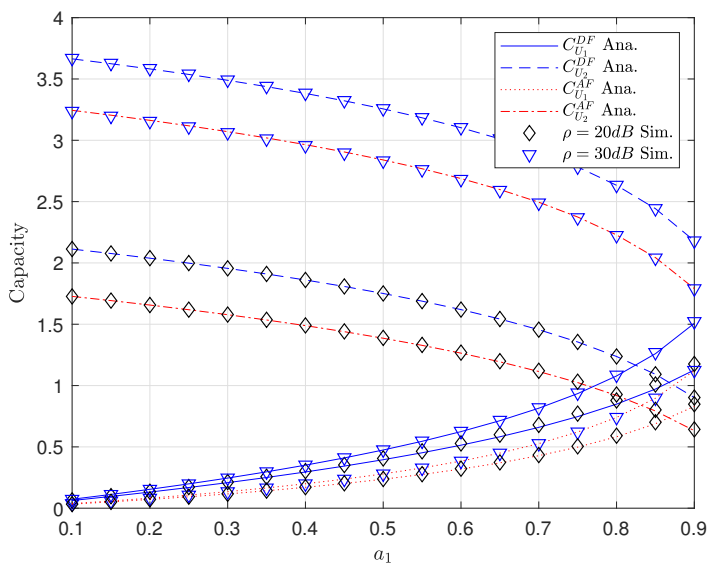

Figure 4. Ergodic capacity performance of two users versus $a_{1}=0.5$ 


\section{CONCLUSION}

This paper considers ergodic capacity of wireless powered network. It can be concluded that power allocation coefficients for each user in EH-NOMA leads to varying ergodic city performance. The detail performance analysis has been performed in term of ergodic capacity and it is provided via the closed-form expressions related to this metric. It is shown that performance gap exists among two NOMA users due to different mode of AF/DF, and different power allocation factors. Simulation results are performed to verify our analytical results and it is helpful guidelines to design EH-NOMA in practice.

\section{REFERENCES}

[1] Q. Zhang, Z. Liang, Q. Li, and J. Qin, "Buffer-aided non-orthogonal multiple access relaying systems in Rayleigh fading channels," IEEE Trans. Commun., vol. 65, no. 1, pp. 95-106, Jan. 2017.

[2] G. Cai, Y. Fang, G. Han, J. Xu, and G. Chen, "Design and analysis of relay-selection strategies for two-way relay network-coded DCSK systems," IEEE Trans. Veh. Technol., vol. 67, no. 2, pp. 1258-1271, Feb. 2018.

[3] M. F. Kader and S. Y. Shin, "Cooperative relaying using space-time block coded non-orthogonal multiple access," IEEE Trans. Veh. Technol., vol. 66, no. 7, pp. 5894-5903, Jul. 2017.

[4] Z. Yu, C. Zhai, and J. Liu, "Non-orthogonal multiple access relaying with truncated ARQ," IET Commun., vol. 11, no. 4, pp. 514-521, Mar. 2017.

[5] P.-M. Nam, D.-T. Do, T.-T. Nguyen, P. T. Tin, "Energy harvesting assisted cognitive radio: random location-based transceivers scheme and performance analysis, “Telecommunication Systems, vol. 67, no. 1, pp. 123-132, 2018

[6] H.-S. Nguyen, D.-T. Do, T.-S. Nguyen and M. Voznak, "Exploiting hybrid time switching-based and power splittingbased relaying protocol in wireless powered communication networks with outdated channel state information," $A u$ tomatika, 58:4, 111-118, 2017.

[7] D.-T. Do, "Energy-aware two-way relaying networks under imperfect hardware: optimal throughput design and analysis," Telecommunication Systems, vol. 62, no. 2, pp. 449-459, 2016.

[8] Quang Nhat Le, Dinh-Thuan Do, Beongku An, "Secure wireless powered relaying networks: Energy harvesting policies and performance analysis," International Journal of Communication Systems, vol. 30, no. 18, 2017.

[9] D.-T. Do, A.-T. Le and B.-M. Lee, "On Performance Analysis of Underlay Cognitive Radio-Aware Hybrid OMA/NOMA Networks with Imperfect CSI," Electronics, vol. 8 no.7, pp. 819, 2019.

[10] Dinh-Thuan Do, Chi-Bao Le, A.-T. Le, "Cooperative underlay cognitive radio assisted NOMA: secondary network improvement and outage performance," TELKOMNIKA (Telecommunication, Computing, Electronics and Control), vol. 17, no. 5, pp. 2147-2154, 2019.

[11] Dinh-Thuan Do, T.-T. Thi Nguyen, "Exact Outage Performance Analysis of Amplify-and-Forward-Aware Cooperative NOMA," TELKOMNIKA (Telecommunication, Computing, Electronics and Control), vol. 16, no. 5, pp. 19661973, 2018

[12] Dinh-Thuan Do, C.-B. Le, "Exploiting Outage Performance of Wireless Powered NOMA," TELKOMNIKA (Telecommunication, Computing, Electronics and Control), vol. 16, no. 5, pp. 1907-1917, 2018.

[13] D.-T. Do, A.-T. Le, C.-B. Le and B. M. Lee "On Exact Outage and Throughput Performance of Cognitive Radio based Non-Orthogonal Multiple Access Networks With and Without D2D Link," Sensors (Basel), vol. 19, no. 15, pp. 3314, 2019.

[14] Dinh-Thuan Do, M.-S. Van Nguyen, T.-A. Hoang and M. Voznak, "NOMA-Assisted Multiple Access Scheme for IoT Deployment: Relay Selection Model and Secrecy Performance Improvement," Sensors, vol. 19, no. 3, p736, 2019.

[15] D.-T. Do and A.-T. Le, "NOMA based cognitive relaying: Transceiver hardware impairments, relay selection policies and outage performance comparison," Computer Communications, vol. 146, pp. 144-154, 2019.

[16] T.-L. Nguyen and Dinh-Thuan Do, "Power allocation schemes for wireless powered NOMA systems with imperfect CSI: An application in multiple antenna-based relay," International Journal of Communication Systems, vol. 31, no. 15, e3789, 2018.

[17] D.-T. Do, M. Vaezi and T.-L. Nguyen, "Wireless Powered Cooperative Relaying using NOMA with Imperfect CSI," in Proc. of IEEE Globecom Workshops (GC Wkshps), Abu Dhabi, UAE, pp. 1-6, 2018.

[18] Dinh-Thuan Do and M.-S. Van Nguyen, "Device-to-device transmission modes in NOMA network with and without Wireless Power Transfer," Computer Communications, Vol. 139, pp. 67-77, May 2019.

[19] T.-L. Nguyen and Dinh-Thuan Do, "Exploiting Impacts of Intercell Interference on SWIPT-assisted Non-orthogonal Multiple Access," Wireless Communications and Mobile Computing, pp. 12, 2018.

[20] E. Khorov, A. Kureev, and I. Levitsky, "NOMA testbed on Wi-Fi," in Proc. IEEE 29th Annu. Int. Symp. Pers., Indoor Mobile Radio Commun.(PIMRC), Sep. 2018, pp. 1153-1154.

[21] J. Zeng, T. Lv, R. P. Liu, X. Su, M. Peng, C. Wang, and J. Mei, ”Investigation on evolving single-carrier NOMA into multi-carrier NOMA in 5G," IEEE Access, vol. 6, pp. 48268-48288, 2018.

[22] F. Kara and H. Kaya, "On the error performance of cooperative-NOMA with statistical CSIT," IEEE Commun. Lett., 
vol. 23, no. 1, pp. 128-131, Jan. 2019.

[23] O. Abbasi, A. Ebrahimi, and N. Mokari, "NOMA inspired cooperative relaying system using an AF relay," IEEE Wireless Commun. Lett., vol. 8, no. 1, pp. 261-264, Feb. 2019.

[24] S. K. Zaidi, S. F. Hasan, and X. Gui, "Evaluating the ergodic rate in SWIPT-aided hybrid NOMA," IEEE Commun. Lett., vol. 22, no. 9, pp. 1870-1873, Jun. 2018.

[25] J. W. Kim, S. Y. Shin, and V. C. M. Leung, "Performance enhancement of downlink NOMA by combination with GSSK," IEEE Wireless Commun. Lett., vol. 7, no. 5, pp. 860-863, Oct. 2018.

[26] J. W. Kim, S. Y. Shin, and V. C. M. Leung, "Performance enhancement of downlink NOMA by combination with GSSK," IEEE Wireless Commun. Lett., vol. 7, no. 5, pp. 860-863, Oct. 2018.

[27] M. Ashraf, J. Jang, J. Han and K. G. Lee, "Capacity Maximizing Adaptive Power Splitting Protocol for Cooperative Energy Harvesting Communication Systems," IEEE Commun. Letters, vol. 22, no. 5, pp. 902-905, May 2018. 